\title{
Design and Implementation of Intelligent Energy Efficient Conveyor System Model Based on Variable Speed Drive Control and Physical Modeling
}

\author{
Irfan Ahmed Halepoto ${ }^{1}$, Muhammad Zakir Shaikh ${ }^{1}$, Bhawani Shankar Chowdhry ${ }^{1}$ \\ and Muhammad Aslam Uqaili ${ }^{2}$ \\ ${ }^{1}$ Department of Electronic Engineering, Mehran University of Engineering \& \\ Technology, Jamshoro, Pakistan \\ ${ }^{2}$ Department of Electrical Engineering, Mehran University of Engineering \& \\ Technology, Jamshoro, Pakistan \\ Corresponding e-mail: irfan.halepoto@gmail.com
}

\begin{abstract}
The technological advancements in process monitoring, control and industrial automation have played a decisive role in increasing the industrial productivity and manufacturing at faster pace even than been dreamed. An ultimatum requirement is an accurate and precise data acquisition mechanism which can be executed through diverse sensors especially for collecting, analyzing and sorting the objects and elements. In this work, an energy efficient conveyor system model is proposed and implemented which not only recognizes and sorts the objects by sensing its colour and place these objects to its destination by using Robotic vehicle but also smartly adjusts the speed of conveyor belts by recognizing the weight of object(s). The variable speed drive (VSD) based an optimal belt speed control mechanism is proposed by smartly sensing the object weight and optimally adjusting the belt speed. The proposed system optimally switches the conveyor system to on/idleloff status to minimize the energy consumption of conveyor belts.

In this work, a mathematical model of the energy efficient conveyor system is also derived by considering the different dynamic parameters. When conveyor belt is fully loaded with objects, the belt moves around with its maximum potential speed, but when conveyor belt is partially or marginally loaded or unloaded, the speed of belt is adjusted accordingly. In this way, a significant amount of energy and cost of energy can be saved. It is anticipated that, the developed intelligent energy efficient conveyor system model will not only modernize the industrial manufacturing and distribution process but will significantly reduce the energy consumption and cost and will lead to increase the life time cycle of conveyor belts.
\end{abstract}

Keywords: Conveyor System, Object Identification, Robotic Vehicles

\section{Introduction}

In any industrial processing plant, the ultimatum objective is to produce standard and high quality products and sell them at prices which make profits. These objectives can be achieved by efficiently designed and optimally controlled processing plants. A process is set of equipment and materials specifically related to some manufacturing operations or system mechanism. The process control is the system of gathering the system information, quantifying the state of the process, standardizing the rules and guidelines to actuate the control elements accordingly [1]. For any process control system, such actions can be executed manually where only humans are involved or in a semi-automatic way where both machines and humans both are mutually involved or even without any 
involvement of human to perform such actions in automatic way by only using machines [2]. Different types of the products being manufactured by an automation process need to be identified, transport and store at ultimate destination locations. For such a process requires an object identifier and sorting system to recognize, classify and sort the different types of manufactured products.

Different types of conveyor systems are in practice for multi objectives and applications specifically for transportation of objects and luggage's from last 50 years. Such conveyor systems are mostly driven by variable speed electric motors or similar type drives to move the parts and objects. The use of universal conveyor belts for moving, loading or unloading and transportation of objects is in practice for many years. The Vanderlande Industries of Belgian city uses the conveyor belts for object sorting applications especially for transportation and courier services [3]. The objects are sorted through barcode reading and according to weight dimensions [4]. Similarly the Track \& Trace service provided by De Post -La Poste also employs barcode and weight mechanism to generate the item based billing for certain customers [5]. Vanderlande Industry also sorts the different types of parcels [6] with weights and dimensions from 50 grams up to $30 \mathrm{~kg}$ and sizes varying up to $900 \times 600$ x $600 \mathrm{~mm}$ [7]. All these Industry uses weight and barcode mechanism to track and sort the objects, they does not possess any colour sensing mechanism to identify and sort the objects. The LEGO kit made by United States of America has the capacity to identify the object boxes by its colour coding [8], but it is an off shelf board kit. Apart from industries, food bakeries and pizza shops also frequently uses a slow-moving chain based conveyor belt to transport food products through an oven within limited defined distance. At the airports the conveyor belts are main tool to transfer passenger's luggage. Traditionally, Bar Code readers are used to classify and sort the objects, according to size, shape, weight, and so on [9]. The data acquisition through sensors involves measurement, adjustment and control of different parameters such as direction, speed, angle etc.

In this work, an intelligent energy efficient conveyor system model is designed and implemented for object identification and placement system through robotic vehicles. The proposed model not only recognizes the objects by sensing its colour but also place the objects to its desired destination by using industrial luggage vehicle. The operation of proposed conveyor system is made energy efficient by smartly adjusting the speed of conveyor belts by sensing the weight of the objects placed on conveyor belts. If the conveyor belt is fully loaded with the object at upper defined limits, the speed of conveyor belt is set at its full potential or if no object is sensed on conveyor belt, the conveyor belt is turned off automatically. If the conveyor belt is partially or marginally fully loaded, the belt speed is intelligently adjusted accordingly. Thus by smartly adjusting the speed of the belt, the significant energy can be saved.

The rest of paper is proceeds as follows. In section 2, the different units of conveyor system are discussed for object identification and placement of objects at destination using robotic vehicles. In Section 3, the physical and mathematical modeling of intelligent energy efficient conveyor belt system is developed. The work is concluded in Section 4.

\section{Design and Implementation of Conveyor Belts for Object Identification and Placement}

The proposed conveyor system model consists of different units as shown in Figure 1.In the manufacturing unit of proposed model; it is assumed that the different products which are being manufactured in any industry are packed in certain different boxes. 


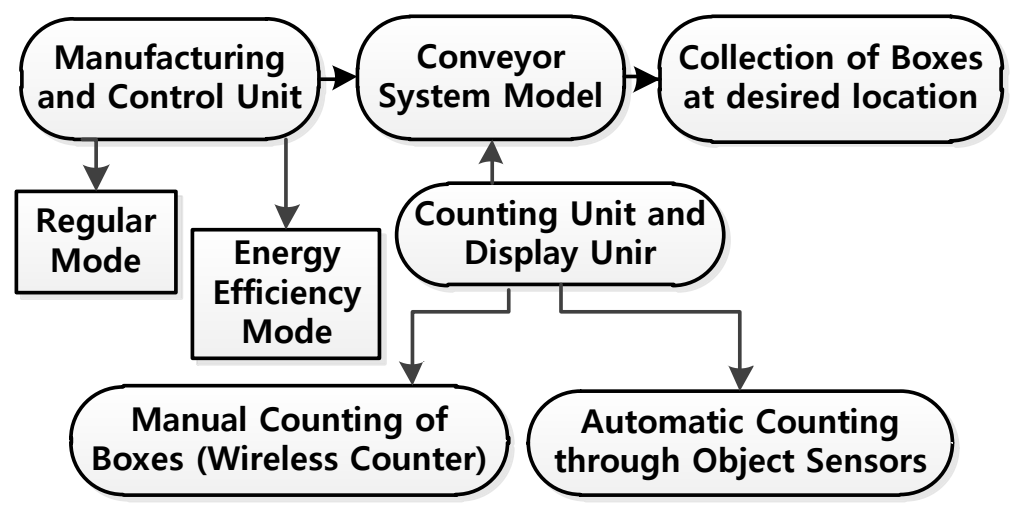

Figure 1. Proposed Conveyor System Model Block Diagram

We have assumed that two separate products are being manufactured and are packed in two different boxes of different colours (i.e. the yellow and green colour boxes) which are need to be delivered at certain desired locations (collection units). In our proposed model, with respect of two object boxes, two conveyor belts are modeled The right sided conveyor belt will be carrying the yellow boxes and left sided conveyor belt is supposed to carry the green boxes. Both yellow and green colour boxes will be moving on the main conveyor belt. Colour sensor is used to differentiate the colour boxes when they reach to colour sensor detection point. At this point the main conveyor system will stop and the colour sensor will sense the colour of the box and it will pass on this information to the controlling unit through the microcontroller. The microcontroller will activate the robotic vehicles to respond accordingly and transport the boxes to the desire destinations. The proposed system process of object manufacturing, object colour identification through sensors and position of conveyor belts and the movement of robotic vehicles are illustrated in Figure 2.

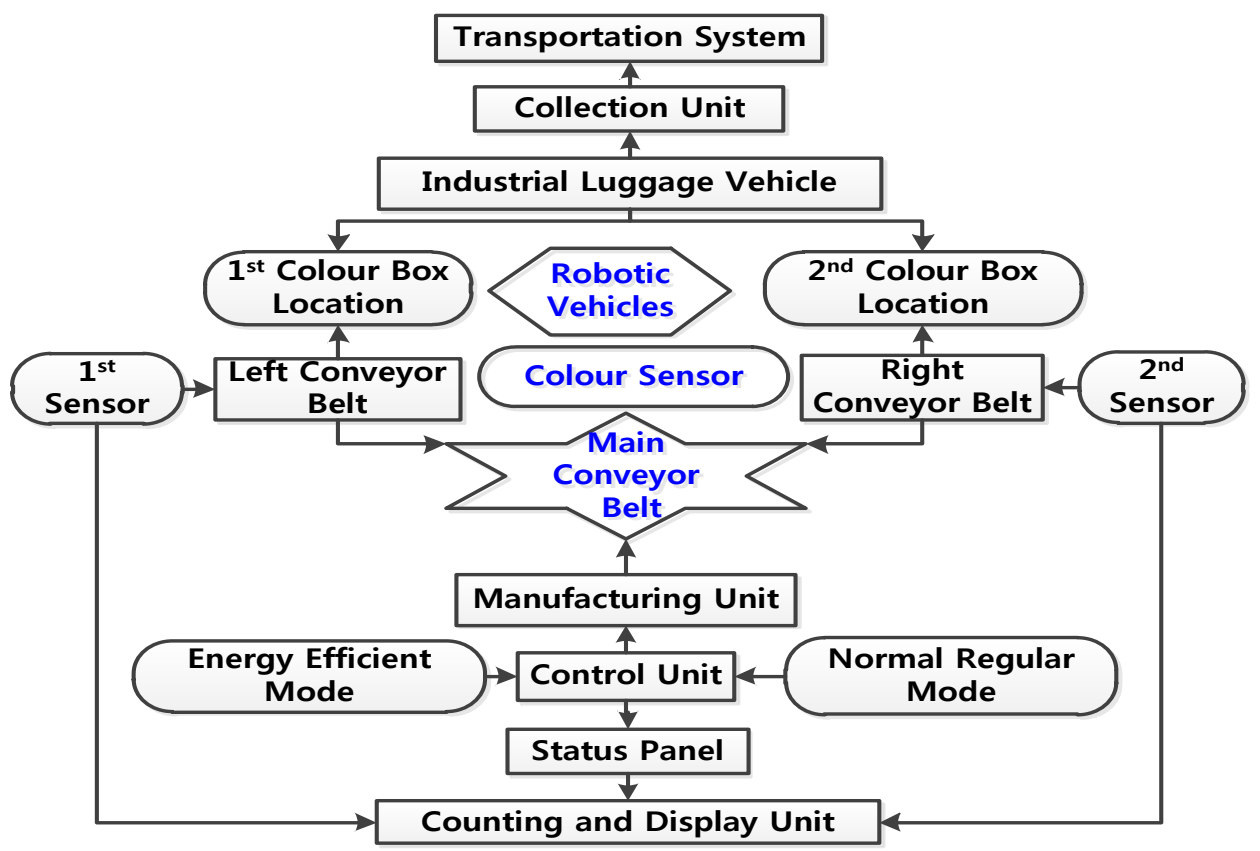

Figure 2. Conveyor System Process 
If the box would be of yellow colour, the robotic vehicles will move anticlockwise to a certain defined degree to move that box on right side of the conveyor belt. Then the conveyor belt will start moving to shift the yellow box to yellow box collection unit. During movement yellow colour boxes towards right side of the conveyor belt, all other belts would remain stationary until the yellow box to reach to its location. After shifting yellow box to its location (yellow box collection unit), the robotic vehicles would restore to its original position. Similarly, for the green box, the same process will occur with the only differences that now the rotation of robotic vehicles would be in clock wise direction; in order to move the green box to the left side of the conveyor belt. This complete process is illustrated in Figure 3.

Once the object is identified through colour sensor and is transported to the desire location or collection point through robotic vehicles, object counting gets started and will be displayed at counting and display panel. Counting and display panel consist of two intelligent sensors that are placed at the right and left side of conveyor belt classified as conveyor belts 2 and 3 to count green and yellow object boxes respectively as shown in Figure 4. The total numbers of object boxes are also shown on display panel. In industries, vehicles, trains, trucks and etc. services are used for the transportation of the manufactured products and objects to be moved from one location to another location [10].

We have designed such a type of indigenous transportation system which are basically two robotic vehicle cars and have named them as luggage vehicles. These luggage vehicles are responsible to transport and dispatch the manufactured objects at destination collection units. The collection units have been classified as different entity points corresponding to the type of object. If the boxes would be of yellow colour, they have to reach at yellow box collection unit. On the other hand, if the boxes are of green colour, they have to reach at green colour box collection unit. This process is shown in Figure 5.

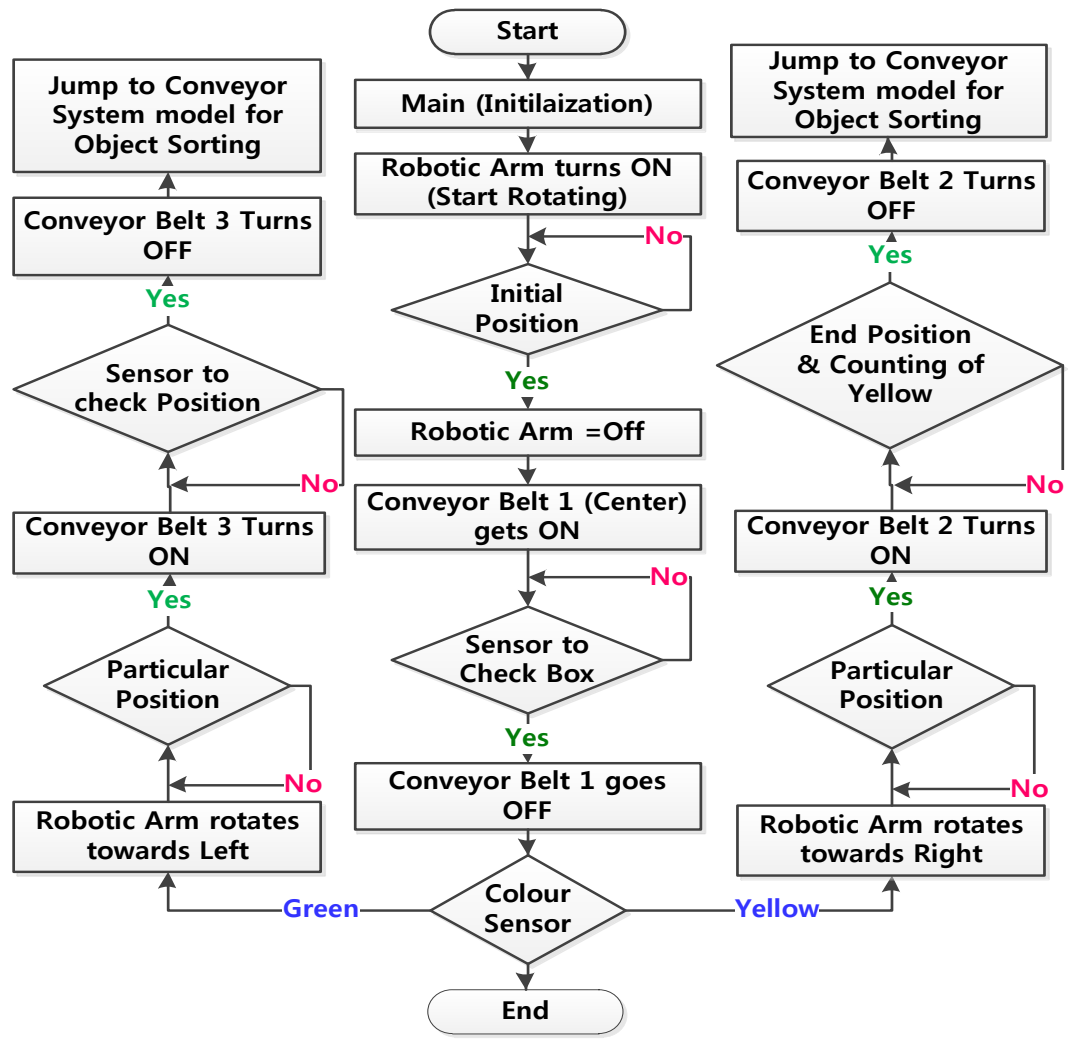

Figure 3. Robotic Vehicles Movement W.R.T to Object Boxes 


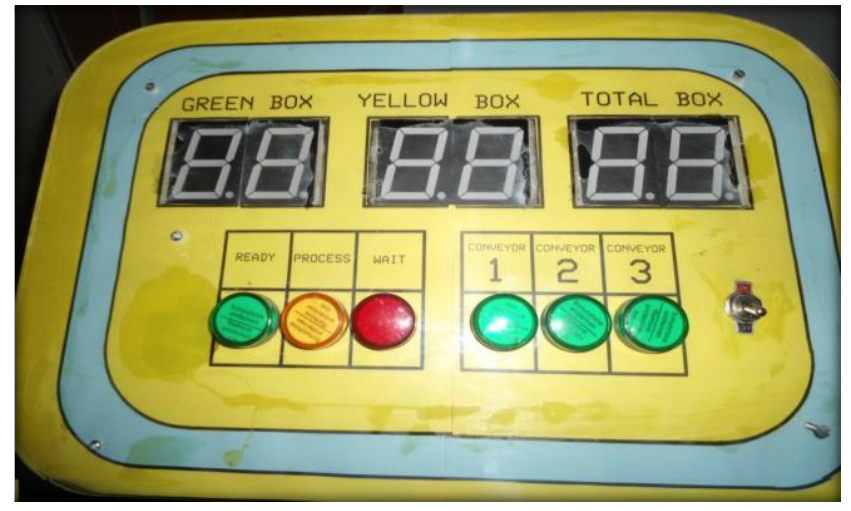

Figure 4. Counting Display Panel for Object Boxes

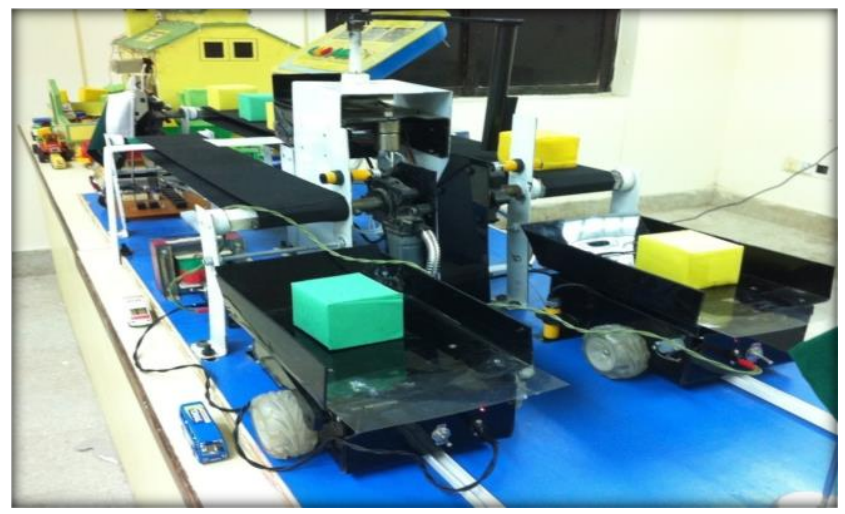

Figure 5. Industrial Luggage Vehicle's For Object Boxes Transportation

\section{Design of Intelligent Energy Efficient Conveyor Belt System}

Energy is an unarguably the critical requirement for the humans to maintain their socio-economic activities [11]. Considering the immense dependency on the energy and the looming global energy crises has compelled the researchers to develop energy efficient systems especially for industry applications. Minimizing the overall power consumption is one of the crucial characteristic of any system design and conveyor systems are no way a different case. Although conveyor systems are proficiently been a preferred way of transporting the small to large scale goods, but energy efficient conveyor system design with optimized power consumption is a significant design requirement for meeting the growing energy demands and eventually making industrial units more profitable. Conveyor Belts are the popular choice for handling the material over limited conveying distances due to their high transportation efficiency compared to other conventional transport methods. Conveyor belt systems normally consume $6 \%$ of total energy consumption [12], so it is significant to reduce handling cost or power consumption. A conveyor belt is a definite energy conversion system from electrical energy to mechanical energy [13]. The most important step to energy efficient design of conveyor system is the design of the energy efficient mechanical conveyor systems. Conveyor belt energy efficiency can be achieved through three components: (i) operational efficiency, (ii) equipment efficiency, and (iii) technological efficiency.

Traditionally, the efficiency of conveyor belt is achieved through designing the efficient equipment and optimally controlling the operational and switching characteristics of equipment. The optimal switching control strategy optimizes the on/off status of the conveyor belt to adjust the power consumption. Such designing only 
considers the equipment operational efficiency characteristics such as idler, belt and drive system for discrete conveyor system. The scope of this work is to improve overall performance efficiency of conveyor system by integrating the operational, equipment, technological components to achieve the objectives of energy consumption efficient and energy cost minimization. Normally, a conveyor belt runs more or less at the same speed whether it is fully loaded or unloaded. If conveyor belts left running when under no load, it consumes as much as $40 \%$ of full load power, which wastes a significant amount of energy. It is therefore important to monitor the load on the belt by using a weight sensing frame or a volume measurement system and adjust the belt speed in such a way that the belt is always running 'full' in terms of volumetric capacity. For this, a certain threshold value for the loading and unloading degree can be is set. For example $90 \%$ and $10 \%$ of conveyor belt loading can be set to adjust belt speed at its full potential and lower possible speed respectively. Figure 6 illustrate the conveyor system speed adjustment as per loading and unloading conditions.

Power savings can be principally achieved by either reducing the friction in the belt conveyor or by optimising system logistic control. The friction in a belt conveyor can be decreased by applying low loss rubber compound in the belt or by using special low loss idler rolls and skirt boards. Power savings can further be achieved by optimising the logistic control of the system by adjusting and controlling the speed of conveyor belts in relationship to full load to no load on the belt feed and belt speed.

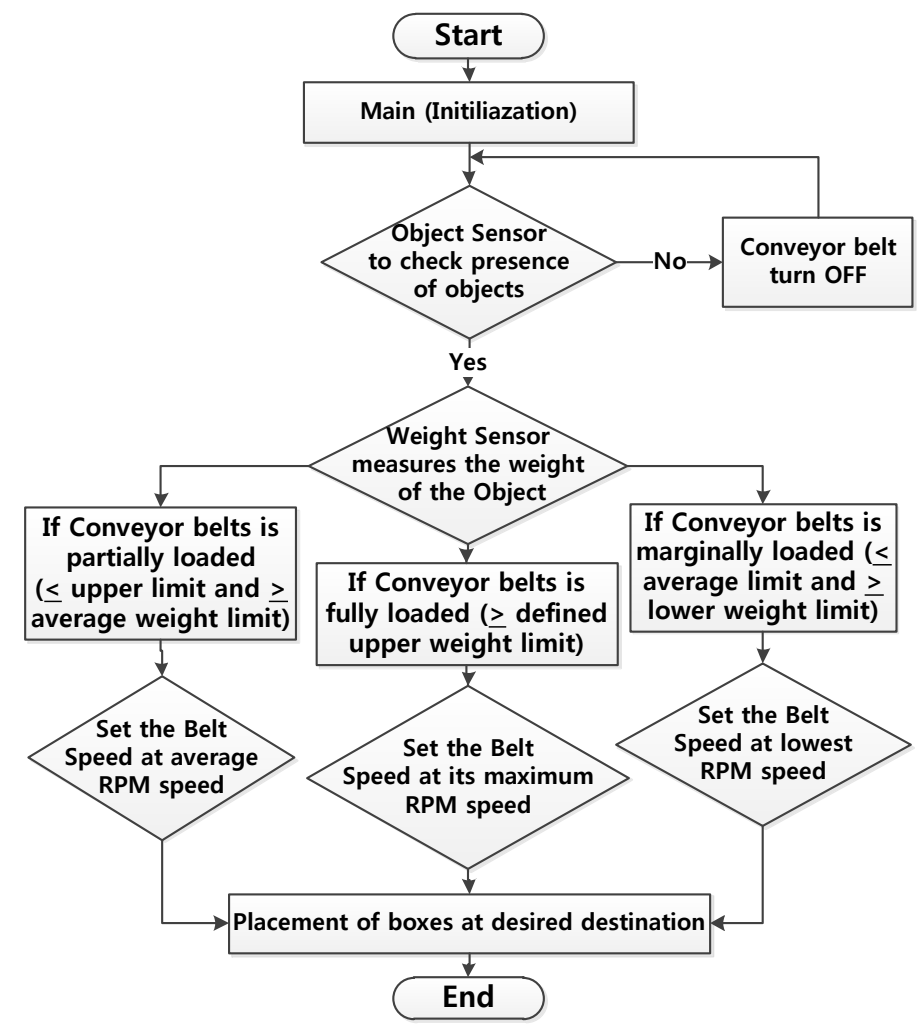

Figure 6. Conveyor Belt Speed Adjusting Mechanism

The belt speed can be controlled in a discrete (on/off) or continuous manner. Decreasing the speed of the belt speed will not only minimize the power consumption but also enhance the life cycle of conveyor belt and associated components like idler rolls. In this work, variable speed drive (VSD) based an optimal belt speed control is proposed to reduce the power consumption of conveyor system. For conveyor applications, VSD can 
significantly reduce power consumption by optimising starting and stopping sequences, acceleration and deceleration ramps, belt loading and conveyor speed.

\subsection{Physical Modeling of Energy Efficient Conveyor System}

A typical Conveyer System consists of electrical power supply, Conveyor belt drive system (CBDS), conveyor main body, braking mechanism and controller system. Generally the CBDS consists of DC motor, gears (speed reduction mechanism), teeth's (sprockets), drive shafts and chains. Integration and supplying power to these elements will generate the torque required to drive the Conveyer System. CBDS also possess special purpose units to control the conveyor belt starting or stopping mechanical dynamics, and speed control units to control the conveyor belt velocity. The first step for conveyor system energy efficiency is the analysis of the conveyor operating modes; such a system is illustrated in Figure 7. This analyse will estimate and evaluate the duration and the frequency of the operating and idle timing of conveyor belts.

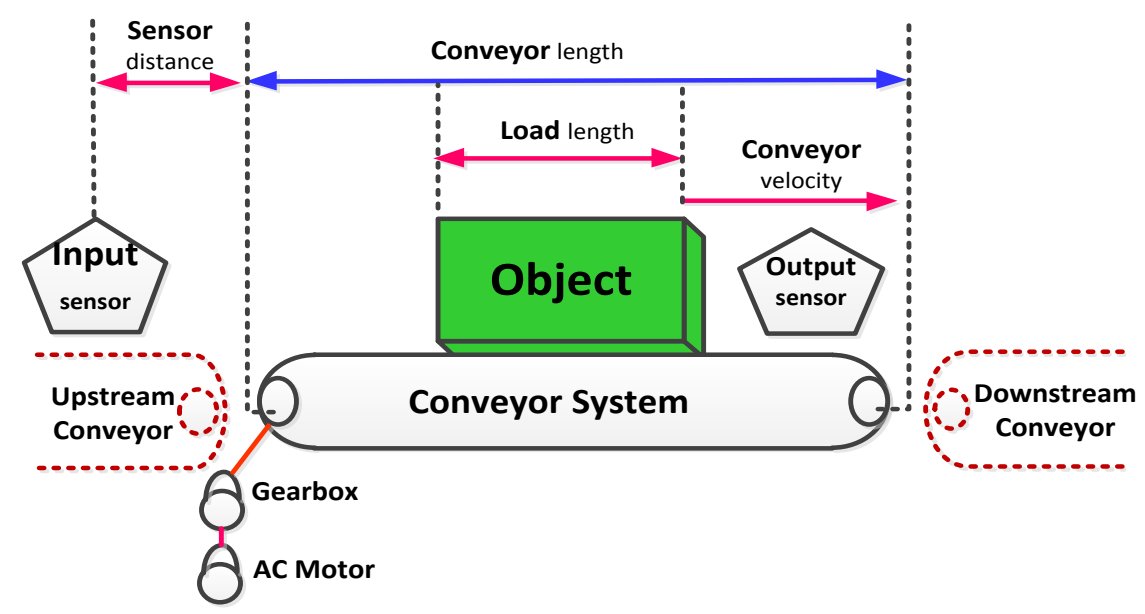

Figure 7. Conveyor System Parameters

The input sensor position is closely related to sensor distance that it covers as a conveyor loading zone must consider following constraints:

and

$$
\text { Sensor }_{\text {distance }}<\text { Load }_{\text {length }}
$$

$$
\text { Sensor }_{\text {distance }} \geq \text { Conveyor }_{\text {velocity }} X \text { starting time }
$$

These two constraints will lead as:

$$
\begin{gathered}
\text { Conveyor }_{\text {velocity } X} \text { starting time } \leq \text { Sensor }_{\text {distance }} \\
<\text { Load }_{\text {length }}
\end{gathered}
$$

Valuing these physical constraints will optimize the energy efficiency of conveyor system.

\subsection{Mathematical Modeling of Energy Efficient Conveyor System}

The electrical power $P_{e}$ needed to drive a conveyor belt depends upon the belt speed $(V)$, aggregate motional resistance $\left(M_{r}\right)$, mechanical efficiency of drive's $\left(\eta_{\text {mech }}\right)$, the frequency converter's electrical efficiencies $\left(\eta_{\text {freq }}\right)$, and the motor efficiency $\left(\eta_{\text {motor }}\right)$. These parameters can be represented as:

$$
P_{e}=\frac{M_{r}\left(m_{L}^{\prime}\right) v}{\eta_{\text {mech }} \eta_{\text {freq }} \eta_{\text {motor }}}
$$


Where, aggregate motional resistance $\left(M_{r}\right)$ depends on conveyor belt load $\left(m_{L}^{\prime}\right)$, its length and conveyor belt design characteristics .

By sensing or measuring the partial or marginal object weight on conveyor belt $\left(Q_{m}\right)$, the belt speed can be reduce which will increases the load on the belt and eventually the motional resistance will increases. As a result of lower belt speed, the power consumption will also reduce accordingly. This is caused by the fact that the increase in friction caused by the increase of belt load $m_{L}^{\prime}$ is less than the decrease in velocity. Therefore $P_{e}$ decreases with a decrease of the belt speed $V$, although not linearly. The speed control savings $\Delta p_{e}$ by lowering the belt speed from $v_{\text {nominal }}$ to $v_{n}$ at non nominal object movement $Q_{m}$ is given as:

$$
\Delta p_{e}\left(Q_{m}\right)=P_{e}\left(Q_{m}, v_{\text {nominal }}\right)-P_{e}\left(Q_{m}, v_{n}\right)
$$

The energy consumption $E_{c}$ can be calculated by multiplying the power required to drive transport equipment with the time $t$ it is operating:

$$
E_{c}=P_{e} t
$$

For a normal conveyor belt $P_{e}$ is a function of time. Sometimes a conveyor belt is fully loaded and is $P_{e}$ high; sometimes it is running empty and accordingly $P_{e}$ is low. Also, the ambient conditions like temperature have an impact on the required drive power. Therefore the total amount of energy used in a certain period of time depends on the operational and ambient conditions and equation (6) can be represented as:

$$
E_{c}=\int P_{e}(t) d t
$$

The equation (7) simplifies conveyor system energy consumption by correlating it with the weight of object at conveyor belt and adjusting the speed of conveyor belt.

\section{Conclusion}

In this work, we have design and implemented an Intelligent Energy Efficient Conveyor System for Object Identification and Placement System using Robotic Vehicles. The proposed model effectively identifies and sorts the objects by using colour sensing approach and transports them towards destination points. Traditionally, the efficiency of conveyor belt is achieved through designing the efficient equipment and optimally controlling the operational and switching characteristics of equipment. The optimal switching control strategy optimizes the on/off status of the conveyor belt to adjust the power consumption. Such designing only considers the equipment operational efficiency characteristics such as idler, belt and drive system for discrete conveyor system.

In this work, to improve the overall performance and efficiency of conveyor system an integrated system of operational, equipment, technological components is proposed and modeled to achieve the objectives of energy efficient and cost minimization conveyor system. The power savings is achieved by optimising the logistic control of the system by adjusting and controlling the speed of conveyor belts in relationship to full load to no load on the belt feed and belt speed. The belt speed is controlled in a discrete switching form. By adjusting the speed of conveyor belt, not only power consumption is reduced but life cycle of conveyor belt and associated components will be also enhanced. It is anticipated that proposed energy efficient conveyor system will help to modernize the manufacturing and services of industrial distribution units. 


\section{Acknowledgment}

This project work was funded by Pakistan Science Foundation under Project No: PSF/Res/S-MUET/ENGG (121). Authors acknowledge the financial assistance provided by Pakistan Science Foundation. Authors are also thankful to Mehran University of Engineering \& Technology, Jamshoro, Pakistan, for providing laboratories and technical support throughout this project.

\section{References}

[1] C. A. Smith and A.B. Corripio, "Principles and practice of automatic process control", Wiley New York, vol. 2, (1985)

[2] C.A. Rosen, "Machine vision and robotics: Industrial requirements", Springer, (1979).

[3] R.Franssen, "Life Cycle Cost Analysis. For Baggage Handling Systems of VanderLande Industries," ed: Technische Universiteit Eindhoven, Eindhoven, Confidential report, (2006).

[4] S,Suzuki, M Uesugi and M Sekine, "Automatic sorting conveyor systems," ed: Google Patents, (1977).

[5] O Herveand, "Device for controlling and regularizing the spacing objects such as parcels, packages," ed: Google Patents, (1992).

[6] J. M. Van Den Goor, "Conveyor with endless conveyor element having interconnected carriers," ed: Google Patents, (1999).

[7] S. J Hammer, "Checkout stand with a barcode reader on a bagging end," ed: Google Patents, (2015).

[8] L,Cole and D.Austin "Visual object recognition using template matching," in Australian conference on robotics and automation, (2004).

[9] R. J, Pang, E. D Barkan and T. E Lackemann, "Method of decoding barcode with imaging scanner having multiple object sensors," ed: Google Patents, (2015).

[10] I. Hussain, I. A. Halepoto, W. Kumar and K. Kazi, "Anti-Slip Traction Control of Railway Vehicle Based on Estimated Wheel-Rail Contact Condition", Sindh University Research Journal (Science Series), vol. 45, no. 2, (2013), pp: 373-378.

[11] I.A Halepoto, M.A.Uqaili and B. S Chowdhry, "Least square regression based integrated multiparameteric demand modeling for short term load forecasting," Mehran University Research Journal of Engineering and Technology, vol. 33, (2014), pp. 215-226.

[12] S. Zhang and X. Xia, "Modeling and energy efficiency optimization of belt conveyors," Applied Energy, vol. 88, (2011),pp. 3061-3071.

[13] S. Zhang and X. Xia, "Optimal control of operation efficiency of belt conveyor systems," Applied Energy, vol. 87, (2010),pp. 1929-1937. 
International Journal of Control and Automation Vol. 9, No. 6 (2016) 